\title{
Alzheimer, mitochondria and gender
}

Amandine Grimm a, b; Ayikoe Guy Mensah-Nyagan c; Anne Eckert a, b, *

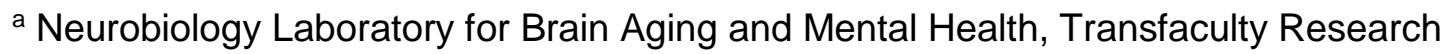

Platform, Molecular \& Cognitive Neuroscience, University of Basel, Wilhelm Klein-Str. 27,

$\mathrm{CH}-4012$ Basel, Switzerland

${ }^{b}$ Psychiatric University Clinics, University of Basel, Wilhelm Klein-Str. 27, CH-4012 Basel,

Switzerland

${ }^{c}$ Biopathologie de la Myéline, Neuroprotection et Stratégies Thérapeutiques, INSERM

U1119, Fédération de Médecine Translationnelle de Strasbourg (FMTS), Université de

Strasbourg, Bâtiment 3 de la Faculté de Médecine, 11 rue Humann, 67000 Strasbourg,

France

* Corresponding Author: Anne Eckert

E-mail: anne.eckert@upkbs.ch

Tel. $+41(0) 613255487$

Fax $+41(0) 613255577$ 


\begin{abstract}
:
Epidemiological studies revealed that two-thirds of Alzheimer's disease (AD) patients are women and the drop of sex steroid hormones after the menopause has been proposed to be one risk factor in AD. Similarly, the decrease of circulating testosterone levels with aging may also increase the risk of AD in men. Studies attest the neuroprotective effects of sex hormones in animal models of $A D$, but clinical trial data remain controversial. Here, we discuss the implication of mitochondria in gender differences observed in AD patients and animal models of $A D$. We summarize the role of mitochondria in aging and $A D$, pointing to the potential correlation between the loss of sex hormones and changes in the brain redox status. We discuss the protective effects of the sex hormones, estradiol, progesterone and testosterone with a specific focus on mitochondrial dysfunction in AD. The understanding of pathological processes linking the loss of sex hormones with mitochondrial dysfunction and mechanisms that initiate the disease onset may open new avenues for the development of gender-specific therapeutic approaches.
\end{abstract}

\title{
Key words:
}

Aging; abnormally hyperphosphorylated tau protein; Alzheimer's disease; amyloid- $\beta$; bioenergetic impairments; gender differences; mitochondria; oxidative stress; sex steroid hormones. 


\section{Introduction}

Brain aging is marked by a gradual decline in energy metabolism coupled with an increased oxidative stress (Yin et al. 2014). Since the brain is a highly specialized organ with significant energy requirements, the age-dependent modifications in cerebral bioenergetic and redox homeostasis may lead to neuronal disturbances and, eventually, neurodegeneration. Brain hypometabolism and oxidative stress are a prominent and early event of Alzheimer's disease (AD) that can be observed even before the appearance of the two histopathological hallmarks of the disease - extracellular amyloid- $\beta(A \beta)$ plaques and intracellular neurofibrillary tangles (NFT) (Perry et al. 2000; Schmitt et al. 2012; Yao et al. 2009). Since the incidence rate for $A D$ increases exponentially after 60 years, especially during the $7^{\text {th }}$ and $8^{\text {th }}$ decades of life, advancing age remains the main risk factor of the disease that currently affects about $2 \%$ of the population in industrialized countries and accounts for more than $60 \%$ of all dementia cases (Prince et al. 2013). With the extended average lifespan, this neuropathology, that is marked by a progressive cognitive and physical decline, will become increasingly burdensome and costly in the coming years as AD prevalence is expected to quadruple by 2050 (Brookmeyer et al. 2007).

Epidemiological studies showed that women represent two-thirds of AD patients and exhibit a greater vulnerability to the disease compared to men (Mielke et al. 2014). These observations were corroborated by animal studies showing that in most of the transgenic $A D$ mouse models, $A \beta$ deposits were more striking in females compared to age-matched males (see details in section 4). Based on these observations, hormonal deficit in post-menopausal women, characterized by a sudden drop in circulating estrogen levels, has been proposed to be another risk factor in AD (Vest and Pike 2013). Numerous evidence highlighted the neuroprotective effect of estrogens in cellular and animal models, in particular, their bearing upon mitochondrial metabolism (Grimm et al. 2012). However, beneficial effects of hormonal replacement therapy (HRT) are still under debate. Similarly, evidence based on human and 
animal studies suggested that androgen deprivation also represents a risk factor for $A D$ pathogenesis in men (Pike et al. 2009; Rosario et al. 2010; Rosario et al. 2011).

Together, these observations indicate that reproductive senescence, especially in women, may represent an additional risk to develop AD. However, the mechanisms underlying sex hormone-dependent neuroprotection are not well understood, nor the implication of mitochondria in this process.

In this review, we summarize the role of mitochondria in brain aging and AD, as well as the link between mitochondrial dysfunction and the loss of sex hormones in both females and males. We give an overview of evidence showing gender differences in the pathogenesis of the disease in both AD patients and animal transgenic models, and further we discuss the protective effects of sex steroid hormones on $A \beta$ peptide and hyperphosphorylated tau-induced neurodegeneration with specific regard to their deleterious effects on mitochondria.

\section{Mitochondrial dysfunction in aging: the beginning of the end in AD?}

Mitochondria play a central role in eukaryotic cell survival and death because they are orchestrating both energy metabolism and apoptotic pathways. They are considered as the "powerhouses of cells", providing the universal cellular fuel via adenosine triphosphate (ATP) generation that is accomplished through oxidative phosphorylation (OXPHOS) from nutritional sources. They contribute to plenty of cellular functions, including apoptosis, cell growth and differentiation, regulation of intracellular calcium homeostasis, alteration of the cellular reduction-oxidation (redox) state and synaptic plasticity. In this context, mitochondria are particularly important in the nervous system that requires for about $20 \%$ of the body's total basal oxygen consumption for neuronal energy support (Shulman et al. 2004). 
Unfortunately, an inevitable by-product of the mitochondrial respiration is the formation of superoxide anion radicals $\left(\mathrm{O}_{2}{ }^{-}-\right)$, mostly by the mitochondrial complexes I and III involved in the electron transport chain (ETC) (Jezek and Hlavata 2005; Turrens 2003). $\mathrm{O}_{2}{ }^{-}-$ can be converted into other reactive oxygen species (ROS) such as hydrogen peroxide $\left(\mathrm{H}_{2} \mathrm{O}_{2}\right)$ and the highly reactive hydroxyl radical $\left(\mathrm{OH}^{*}\right)$ through enzymatic and non-enzymatic reactions (Adam-Vizi and Chinopoulos 2006; Balaban et al. 2005; Turrens 2003). According to the "free-radical theory of aging" proposed in 1956 by Harman (Harman 1956), when ROS are produced in excess, they can induce an oxidative stress, damaging proteins and DNA, and inducing lipid peroxidation, with the corresponding mitochondrial structures as the first targets of toxicity. Especially, long polyunsaturated fatty acid chains of mitochondrial membranes are very susceptible to oxidation and may lead to the membrane depolarization and consecutively to mitochondrial impairments (Harper et al. 2004). In turn, ROS-induced mitochondrial dysfunction may lead to an enhancement of superoxide anion radicals production by the ETC, triggering a "vicious cycle" of oxidative stress (Kalous and Drahota 1996; Lee and Wei 1997; Wallace 2005). As a result, mitochondrial dysfunctions have been implicated in the pathogenesis of neurodegenerative disorders, including $A D$, that are characterized by a cerebral hypometabolism and an impaired homeostasis in the redox status.

Changes in mitochondrial bioenergetics and redox homeostasis are both hallmarks of normal brain aging. We recently summarized the common features observed in both brain aging and $A D$, placing mitochondrial in the center of pathological events that separate normal and pathological aging (Grimm et al. 2016b). In a general manner, a decrease in mitochondrial activity, including OXPHOS, protein level and activity of complexes involved in the ETC, can be observed with increasing age (reviewed in(Grimm et al. 2016b; Leuner et al. 2012a). In parallel, antioxidant defenses are decreased, such as glutathione (GSH) and superoxide dismutase (SOD) activity and/or content, which may result in an increased free- 
radical production and triggers a vicious cycle of oxidative stress leading to neurodegeneration.

A lot of studies support the central role of mitochondria in the early neurodegenerative process occurring in AD (reviewed in (Eckert et al. 2011)). Defects in brain metabolism and increased oxidative stress were found in brains from $A D$ patients and $A D$ transgenic mice even before the appearance of A $\beta$ plaques and NFT (Perry et al. 2000; Schmitt et al. 2012; Yao et al. 2009). The peptide $A \beta$ is generated via the proteolytic cleavage of the amyloid precursor protein (APP) by the enzyme $\beta$-secretase and $\gamma$-secretase, unlike the non amyloidogenic pathway using the a-secretase (LaFerla et al. 2007). NFTs are formed by an aberrant intracellular accumulation of microtubule-associated tau proteins (MAPT) within neurons, resulting of an abnormal tau hyperphosphorylation and its assembly into filaments (Brunden et al. 2009).

Both proteins, $A \beta$ and abnormal tau, were shown to have a direct impact on mitochondrial function (reviewed in (Schmitt et al. 2012)). More specifically, data from cellular and transgenic animal models demonstrated that the presence of $A \beta$ impairs the mitochondrial membrane potential, decreases the mitochondrial complex IV activity and the production of ATP, as well as increases the levels of ROS (Keil et al. 2004; Rhein et al. 2009a; Rhein et al. 2009b). Of note, mitochondria are highly dynamic organelles that continuously fuse and divide in order to maintain a homogeneous mitochondrial population by mixing their content of DNA, lipids and proteins. (Campello and Scorrano 2010). This mitochondrial dynamics (fusion/fission activity) is also impaired in the presence of $A \beta$ indicated by a mainly fragmented mitochondrial network state that might, vice versa, have a negative impact on bioenergetic functions of these organelles (Wang et al. 2008a; Wang et al. 2009; Wang et al. 2008b).

The effect of abnormally hyperphosphorylated tau protein on mitochondrial function has also been investigated (DuBoff et al. 2013; Eckert et al. 2014). First, the microtubules' dissociation induced by tau hyperphosphorylation impairs the transport of distinct cargoes along neuronal axons, including mitochondria (Eckert et al. 2014). In consequence, the 
number of mitochondria transported to the synapse is reduced, which may lead to synaptic degeneration and neuronal death. In addition, the presence of abnormal tau impairs the activity of the mitochondrial complex I in vitro and in vivo (David et al. 2005; Schulz et al. 2012), paralleled by a decreased ATP level and increased ROS production. Like A $\beta$, hyperphosphorylated tau appears to have a negative impact on the mitochondrial dynamics by reducing the fission, which induced an elongation of the mitochondrial network (DuBoff et al. 2012).

Since $A D$ is marked by both $A \beta$ and tau pathologies, both proteins have to be taken into account to dissect the underlying mechanisms of mitochondrial dysfunction. Thus, recent evidence suggests that $A \beta$ and abnormally hyperphosphorylated tau protein may act synergistically to trigger mitochondrial toxicity in AD (Eckert et al. 2011; Eckert et al. 2010; Ittner and Gotz 2011; Schmitt et al. 2012). In short, $A \beta$ appears to trigger a dysfunction primarily of mitochondrial complex IV activity and later also of complex I, whereas the presence of abnormally hyperphosphorylated tau protein impaired complex I activity in the first place (David et al. 2005; Rhein et al. 2009b). Thus, in the presence of both $A \beta$ and abnormal tau, mitochondria exhibit stronger defects in OXPHOS, synthesis of ATP, and reactive oxygen species, emphasizing synergistic, age-associated effects of these two AD hallmarks upon mitochondria (Rhein et al. 2009b).

Together, these findings highlighted the key role of mitochondria in AD pathogenesis. However, the underlying mechanisms leading to the collapse of mitochondrial function are still not understood.

Over the last decade, a growing body of evidence championed the "mitochondrial Alzheimer cascade hypothesis" stated by Swerdlow in 2004 (Swerdlow and Khan 2004). This hypothesis postulates that mitochondrial DNA mutations, accumulating over a person's lifespan, result in mitochondrial dysfunction as a direct cause of aging, generating additional mitochondrial deregulation. This leads to a decrease in energy production and increase in ROS production as well as oxidative stress, which eventually surpasses a functional 
threshold after the failure of compensatory mechanisms and causes neurodegeneration (reviewed in (Swerdlow et al. 2014)).

Accordingly, recent in vitro evidence showed that the elevation of ROS levels by mitochondrial complex I inhibition is able to trigger $A \beta$ production by shifting APP processing towards the amyloidogenic pathway (Leuner et al. 2012b; Stockburger et al. 2014). In line, other studies showed that oxidative stress may lead to an up-regulation of the $\beta$-secretase activity and expression level and consequently to an increased $A \beta$ generation (Guglielmotto et al. 2009; Tamagno et al. 2008; Tamagno et al. 2005).

Taken together, these findings emphasize the key role of mitochondrial redox homeostasis both in aging and in AD. Thus, a disturbed mitochondrial function can lead to the accumulation of ROS. Then, oxidative stress may trigger a circular chain of events including $A \beta$ generation, tau hyperphosphorylation but also neuroinflammation leading to neurodegeneration (reviewed in (Grimm et al. 2016b)).

\section{Normal aging, mitochondrial dysfunction and the loss of sex hormones}

Increasing evidence indicates that the age-dependent changes in bioenergetics and redox homeostasis are subject to gender differences. Indeed, before the menopause, women appear to be better armed to fight against oxidative stress by having higher antioxidant defenses compared to men (Vina and Borras 2010). For instance, in a study monitoring glutathione (GSH) content in the human brain from healthy subjects in vivo, young females ( \pm 26 years old) appeared to have higher GSH levels in the frontal and parietal cortex compared to young men (Mandal et al. 2012). A gradual decrease of this antioxidant enzyme was observed during aging from young to old ( \pm 56 years old) females. In line, the levels of monoamine oxidase $A(M A O-A)$, a relevant source of ROS besides the respiratory chain in the brain, was found to be elevated by $34 \%$ in the brain of perimenopausal women (41-51 years old) compared to younger women (21-40 years old) (Rekkas et al. 2014). A study performed on premenopausal women who underwent bilateral oophorectomy shows that 
estradiol blood levels dropped after the surgery, paralleled by an increase in oxidative stress which was resolved after estrogen replacement therapy (ERT) (Bellanti et al. 2013).

Similar observations were made in animals in which liver and brain mitochondria from young female rats (4-6 months) presented lower peroxide production compared to males of the same age (Borras et al. 2003). In addition, females presented higher antioxidant defenses at this age than males, namely manganese superoxide dismutase (MnSOD), GSH and glutathione peroxidase (GPx) levels. Interestingly, when females were ovariectomized (OVX), mitochondrial peroxide and GSH levels were similar to those of males (Borras et al. 2003; Vina and Borras 2010). This phenomenon was reversed by a treatment with estradiol, the most abundant estrogen in females, highlighting the protective effects of this steroid against oxidative stress. In agreement, another study demonstrated that in OVX mice (6 months old) the level of brain lipid peroxides were increased together with a decrease of the mitochondrial respiratory control ratio $(\mathrm{RCR})$, pyruvate dehydrogenase $(\mathrm{PDH})$ as well as cytochrome c oxidase (COX) activity when compared to sham operated animals (Yao et al. 2012). Again, these disturbances of brain energy metabolism were prevented by a treatment with estradiol.

In perimenopausal rodent models, the drop of estradiol and progesterone concentrations in the brain was accompanied by an increased expression of genes involved in fatty acid oxidation and the ketogenic pathway, a decrease in COX activity and ATP production, and a rise in oxidative stress markers (Yao et al. 2010; Yin et al. 2015). These findings suggest that the metabolic shift from a mitochondrial phenotype towards a ketogenic/fatty acid substrate-dependant phenotype, occurring in perimenopausal age in females, might modulate their sensitivity to oxidative insults.

In men, the loss of steroid sex hormones is less drastic compared to women. The levels of circulating testosterone, the main male sex hormone, present a gradual reduction over the life course, decreasing of approximately $2 \%$ every year after the age of 30 years (Feldman et al. 2002), while brain oxidative stress shows a gradual increase with age in men (Peskind et al. 2014). Moreover, circulating levels of testosterone were positively correlated 
with the level of the antioxidant glutathione S-transferase in a low oxidative stress environment in Caucasian and Mexican-American men (over 60 years old) (Cunningham et al. 2014), while in gonadectomized (GDX) rats, SOD and GSH activity significantly decreased in the hippocampus when compared to that of sham-operated animals (Meydan et al. 2010). Again, these effects were prevented in animals after testosterone administration.

It is important to note that steroids can also be synthesized locally within the nervous system independently of peripheral steroidogenic glands. This specific category of molecules is called "neurosteroids" (Corpechot et al. 1981). Interestingly, the biosynthesis of both peripheral steroids and neurosteroids start within the mitochondria by the transport of cholesterol from the cytosol to the mitochondrial matrix via a protein complex involving the steroidogenic acute regulatory protein (StAR) and the translocator protein (TSPO) (Rone et al. 2009). Once in the mitochondrial matrix, molecules of cholesterol are converted into pregnenolone, the precursor of all steroids, by the cholesterol side-chain cleavage enzyme referred to as cytochrome $\mathrm{P} 450_{\mathrm{scc}}$. This process constitutes the rate limiting step of the steroidogenesis and highlights the paramount role of mitochondria in steroid homeostasis. Thus, one can suggest that the age-related accumulation of mitochondrial deficits may impact the biosynthesis of steroids and constitute a potential pathogenic mechanism leading to neurodegeneration (Velarde 2014).

Of note, the peripheral pool of steroids contributes to the brain pool, but it is still not clear to which extent. Moreover, steroid levels measured in the blood do not necessarily correspond to the brain steroid concentrations. Thus, the advantage of using GDX animals is the measurement of the pure brain pool of neurosteroids, in contrast to the normal situation detecting the total pool of neurosteroids and blood-derived steroids in the brain. In a recent study, Caruso and colleagues (2013) compared neurosteroid levels in the limbic region of young mice ( 7 months old) and old mice (24 months old) (Caruso et al. 2013). They showed an alteration in neurosteroid concentration with a general trend toward lower steroid levels in the brain of aged mice compared to young mice. 
Recently, Melcangi and colleagues summarized sex differences observed in the level of neuroactive steroids (neurosteroids and/or steroid hormones) both under physiological conditions and in different neurodegenerative diseases, including AD (Melcangi et al. 2015). Indeed, sex differences can already be observed at the level of the expression of proteins and enzymes involved in steroidogenesis, such as StAR and $\mathrm{P} 450_{\mathrm{scc}}$, and reflect dissimilar levels of steroids in distinct rodent brain regions of males compared to females (Melcangi et al. 2015). These observations suggest that males and females are not identically sensitive to various nervous system alterations due the sex differences in the level of neuroprotective steroids in different regions of the brain.

Taken together, these findings strongly suggest that there is a tight relationship between the age-related decrease in steroid levels and the age-related increase in oxidative stress (Fig. 1). Since the loss of estrogens is more drastic at the menopause, women may present a higher vulnerability to oxidative stress at this period of time, promoting the onset of neurodegenerative disorders, especially AD.

\section{Impact of gender on $A \beta$ and/or tau pathology}

\subsection{Gender differences in $A D$ patients}

As mentioned in the introduction, epidemiological and observational studies put in evidence a higher prevalence and incidence of $A D$ in women than in age-matched men (Mielke et al. 2014). Gender differences emerged from different studies that focused on diverse aspects of the disease. For instance, antioxidant defenses, more specifically SOD and GPx activity, were partially upregulated in the brain of female AD patients, possibly as a compensatory mechanism against oxidative stress (Schuessel et al. 2004). In parallel, oxidative damage measured by the levels of 4-hydroxynonenal (HNE) were higher in women than in men ( \pm 77.3 years old), indicating that female AD patients are more sensitive to 
oxidative stress. In line, an in vivo evaluation of GSH content showed that brain GSH levels were further depleted in female $\mathrm{MCl}$ (mild cognitive impairments) and $\mathrm{AD}$ patients (Mandal et al. 2012).

On a histopathological point of view, women exhibit a more extensive $A \beta$ deposition at early stages of NFT formation compared to men (Corder et al. 2004). Women appear to have a more global AD pathology due to more NFT (Barnes et al. 2005). Cerebral atrophy is also a subject to gender differences, with female $\mathrm{MCl}$ and $\mathrm{AD}$ patients having a faster decrease in brain volume (Skup et al. 2011). Gender differences in cognitive abilities as well as impairments remain a controversial topic; however a faster rate of cognitive and functional decline was noted in women (Mielke et al. 2014). These effects are even more pronounced in female AD patients carrying the $\varepsilon 4$ allele of the apolipoprotein $\varepsilon(A P O E \varepsilon 4)$ that represent the main genetic risk factor for sporadic AD (Reitz and Mayeux 2014). Indeed, there is a fourfold increased risk to develop $A D$ in women with one $\varepsilon 4$ allele compared to men who present only a little increased risk (reviewed in (Mielke et al. 2014)). Furthermore, female $\varepsilon 4$ carriers presented the strongest cognitive impairment, connectivity changes and brain atrophy as well as the highest load of $A \beta$ plaques and NFT.

\subsection{Gender differences in animal models of amyloidopathies}

These observational studies performed in humans are corroborated by a broad range of evidence emerging from transgenic animal models. Indeed, consistent findings were obtained by using simple, double and even triple transgenic AD mouse models, indicating a higher $A \beta$ load in female than in age-matched male brains (Fig. 2).

The use of animal models of AD was made possible by the identification of autosomal dominant mutations in familial cases of $A D(F A D)$, representing less than $1 \%$ of the total number of $A D$ cases, and are characterized by an early disease onset at an age younger than 60 years (Reitz and Mayeux 2014). Mutations in three genes are now identified: i) the gene coding for the amyloid precursor protein (APP, more than 20 pathogenic mutations 
identified); and genes coding for ii) the presenilin 1 (PS1) and iii) presenilin 2 (PS2) (over 130 mutations identified) (Götz and Ittner 2008; Van Dam and De Deyn 2006). These three genes are directly linked to the accumulation of $A \beta$ deposits in the brain due to the increased production of $A \beta$ peptide from its precursor protein APP, suggesting a direct and pathological role for $A \beta$ accumulation in the development of $A D$.

In transgenic mice bearing mutations in APP or APP/PS1, and thus leading to an amyloidopathy, constant findings showed that female mice present an $A \beta$ accumulation and $A \beta$ deposits at earlier ages compared to males (Fig. 2). Sex-differences were also observed in the development of cognitive declines. Indeed, deficits in learning and memory were more precocious and pronounced in female APP and APP/PS1 mice (Callahan et al. 2001; King et al. 1999). In parallel to the $A \beta$ accumulation and cognitive dysfunction, female mice (wildtype and transgenic APP/PS1) exhibited increased microglial activation, an increase in the A $\beta$ producing enzyme BACE-1 ( $\beta$-secretase 1) and a decrease in the insulin-degrading enzyme (IDE), an A $\beta$ clearance factor (Gallagher et al. 2013). Similar observations were made in 5xFAD mice bearing the APP Swedish mutation (K670N, M671L), the Florida mutation (I716V), the London mutation (V717I), and PS1 mutations (M146L, L286V) (Devi et al. 2010). In this model, it was shown that $A \beta$ levels were significantly increased in the hippocampus of female mice after 5 days of restraint stress, coupled with an increase of BACE-1 protein and mRNA levels, full-length APP expression and phosphorylation of eukaryotic initiation factor-2a (elF2a, involved in the post-transcriptional upregulation of BACE1). These increases were not observed in stressed males, suggesting an exquisite vulnerability of the female brain, and especially the female hippocampus, towards stress, additionally favoring the production of $A \beta$ via the amyloidogenic pathway of APP processing.

Gender-specific changes were also detected in the metabolomic profile of APP/PS1 transgenic mouse brains, with females presenting greater metabolic changes than males (Trushina et al. 2012). Furthermore, the hydrogen peroxide $\left(\mathrm{H}_{2} \mathrm{O}_{2}\right)$ production as well as cytochrome c release, an initiator of cellular apoptosis, were enhanced in isolated brain mitochondria from old female rats after incubation with $A \beta$ peptide ( 6 hours, $10 \mathrm{mM}$ ), but not 
in mitochondria from young females (Vina et al. 2007). Of note, the level of cytochrome c release detected in old females after treatment with $A \beta$ was similar to that measured in mitochondria from young males, indicating that young females are particularly resistant to $A \beta$ toxicity, probably due to a sex-specific enhanced antioxidant defense system (Borras et al. 2003), suggesting that the loss of sex hormones with increasing age makes females more vulnerable to $A \beta$ toxicity.

\subsection{Gender differences in animal models of tauopathies}

Since the second main hallmark of $A D$ is the presence of abnormally hyperphosphorylated tau protein aggregated in NFTs, one can expect that mutations in the tau coding gene may be involved in the pathogenesis of early onset AD cases. But surprisingly, no mutations in microtubule associated protein tau have been so far detected in $F A D$, but have been identified in familial frontotemporal dementia with Parkinsonism linked to chromosome 17 (FTDP-17) (Goedert and Jakes 2005) leading to NFTs formation.

Thus, the identification of these mutations in the tau protein allowed the elaboration of animal models of tauopathies (Götz and Ittner 2008). The investigation of sex-differences in the development of NFTs and cognitive deficits has revealed different outcomes in function of the models used (Fig. 2). For instance, no differences between males and females have been observed in pR5 mice bearing the tau P301L mutation (longest four-repeat tau 4R2N, Thy1 promoter), with regard to their rate of mortality or other tau-related characteristics (Kremer et al. 2011). However, in JNPL3 mice bearing the shortest four-repeat 4R0N human tau with the P301L mutation (PrP promoter), females displayed more insoluble tau than males at 3, 6 and 9.5-11 months of age (Lewis et al. 2001). Similarly, in rTg4510 mice, an inducible transgenic model of mice bearing the P301L mutation, females displayed more tau aggregates in the brain as well as accumulation of abnormal tau in the cerebrospinal fluid (CSF) compared to males (Song et al. 2015). Levels of tau hyperphosphorylation were also 
associated with more serious cognitive impairments in females, namely learning and memory deficits (Yue et al. 2011).

In contrast, in mice bearing the P301S mutation (human tau 4R/1N, PrP promoter), male presented a more striking mitochondrial proteome deregulation (impairments in enzymatic activity of citrate synthase, complex I, ATP synthase, aconitase and glutathione reductase) at 10 months of age compared to females (Dumont et al. 2011). Increased levels of tau phosphorylation were only found in males at 7 and 10 months of age.

Together, these findings indicate that gender-differences observed in animal models of tauopathies seem to be dependent of the mutation and/or the promoter (Thy1 or PrP) which was used to express the transgenes. Further studies are needed to unravel in more details the underlying mechanisms.

\subsection{Interaction of $A \beta$ and tau}

Crossing animal models of amyloidopathies with those of tauopathies allowed the investigation of the contribution of both AD-related proteins on the pathogenesis of the disease (Fig. 2). Similar to mice models of amyloidopathies, consistent findings showed that females bearing mutations in APP and tau are more affected by the disease than males. For instance, in a study investigating the development of NFTs in TAPP mice (double transgenic: JNPL3 x Tg2576), females presented greater amounts of insoluble tau compared to males, reflecting a similar sensitivity as already observed in single transgenic animals (JNPL3) (Lewis et al. 2001). An enhanced NFT pathology was also detected in TAPP females in comparison to single transgenic (JNPL3) ones. These findings are in agreement with previous data showing that the presence of $A \beta$ triggers NFTs formation (Eckert et al. 2008; Götz et al. 2001). Thus, one can speculate that the higher $A \beta$ levels found in female Tg2576 mice can exacerbate NFT pathology especially in female TAPP animals.

In two models of triple transgenic AD mice (3xTgAD and Triple AD mice) (Fig. 2), females presented a greater $A \beta$ burden compared to males ((Carroll et al. 2010a; Hirata- 
Fukae et al. 2008), and (Grueninger et al. 2010): with respect to gender differences: unpublished observations). An increase in $\beta$-secretase activity was already observed in 9 months old 3xTgAD females compared to age-matched males, followed by a decreased level of neprilysin, an $A \beta$ degrading enzyme, at 16 and 23 months (Hirata-Fukae et al. 2008). However, no gender-differences in tau pathology were detected in 3xTgAD mice.

Taken together, these findings suggest that $A \beta$ burden is the foremost hallmark in females, thus favoring the amyloidogenic pathway rather than the tau pathway, in the development of $A D$ in women. However, the molecular mechanisms through which clinicallyrelevant precipitating factors contribute to the sex-specific pathophysiology need to be elucidated and the drastic change in female sex hormones in menopause might be one of them.

\section{Protective effects of sex hormones against $A \beta$ and tau burden}

Given the known gender differences in AD-related cognitive decline and accumulation of $A \beta$ and NFTs, one can hypothesize that the age-related decrease in sex steroid hormones plays a role in the pathogenesis of the disease due to the loss of their protective actions. To this regard, many cellular and animal studies examined the effects of sex steroids against $A \beta$ and tau toxicity.

\subsection{Effects on $A \beta$ pathology}

Since the sudden drop in estrogen levels after the menopause has been proposed to be one risk factor in $A D$, estrogen replacement protocols have been considered to alleviate the loss of this hormone in females.

Animal studies showed that a treatment with estradiol induced a decrease in $A \beta$ levels and its aggregation into plaques in female mice expressing human APP bearing the Swedish and Indiana mutations compared to wild-type mice (Amtul et al. 2010). The 
underlying mechanisms are still poorly understood but one possible mechanism seems to be the shifting of APP processing towards the non-amyloidogenic pathway ( $\alpha$-secretase pathway) via activation of extracellular-regulated kinase 1 and 2 (ERK 1 and 2) and through protein kinase C (PKC) signaling pathway by estrogens (Pike et al. 2009). Of note, studies showed that the activation of ERK1/2 also inhibits the $\mathrm{Y}$-secretase activity, leading to a decreased $\beta$-secretase (BACE1) expression and activity, and thus a decreased level of $A \beta$ (Tamagno et al. 2008; Tamagno et al. 2009). Since BACE1 activity appears to be enhanced by oxidative stress (Leuner et al. 2012b; Tamagno et al. 2008), another possible mechanism would be that estradiol may activate $\mathrm{ERK} 1 / 2$ as well as antioxidant defenses, leading to an inhibition of $\gamma$-secretase and BACE1, and a decreased $A \beta$ production.

In line with these studies using estradiol treatment, a depletion of sex steroid hormones, induced by OVX in 3xTgAD female mice, vice versa significantly enhanced $A \beta$ accumulation and had a negative impact on cognitive performances (Carroll et al. 2007; Frye and Walf 2008; Yao et al. 2012). Of note, those effects were prevented by a treatment with estradiol.

To study the implication of the brain pool versus circulating levels of estrogen on $A D$ pathology, Yue and colleagues have crossed APP23 mice with estrogen-synthesizing enzyme aromatase gene knockout mice (Yue et al. 2005). They showed that these mice presented very low brain estrogen levels, an early-onset $A \beta$ accumulation as well as greater $A \beta$ burden and impaired $A \beta$ clearance and degradation when compared to APP23 mice and APP23 OVX mice. In line, inhibition of aromatase activity using anastrozole significantly increased $A \beta$ deposits in the hippocampus of 3xTgAD female mice (Overk et al. 2012).

Taken together, these findings put in evidence the implication of brain estradiol metabolism in the development of $A \beta$ pathology.

The effects of progesterone, which is the second main steroid hormone in female, on $\mathrm{A} \beta$ pathology are less investigated. In a study combining in vitro and in vivo approaches, progesterone and estradiol increased the expression of $A \beta$ clearance factors, at least in part 
via steroid receptor activation (Jayaraman et al. 2012). Interestingly, continuous progesterone treatment, alone or in combination with estradiol, did not reduce $A \beta$ accumulation and attenuated the effects of estradiol in female OVX 3xTgAD mice (Carroll et al. 2007; Carroll et al. 2010b). However, cyclic progesterone administration lowered $A \beta$ load and enhanced $A \beta$-reducing effects of estradiol during a co-treatment with both steroids (Carroll et al. 2010b). This data are in line with the cyclic nature of female sex hormone levels along the estrous cycle. Thus, the timing and duration of hormone administration should be taken into account if a treatment combining both estradiol and progesterone is considered.

Mounting evidence coming from human and animal studies suggested that androgen deprivation represents a risk factor for AD pathogenesis in males (Pike et al. 2009; Rosario et al. 2010). In men with "mild neuropathological changes", brain testosterone levels were inversely correlated to soluble $A \beta$ concentration (Rosario et al. 2011). In a recent study, Overk and colleagues (Overk et al. 2013) examined basal levels of serum and brain testosterone in male 3xTgAD mice and found that brain testosterone levels rise with disease progression. This increase in testosterone in aged male 3xTgAD mice was correlated with reduced $A \beta$ plaque pathology, suggesting an implication of testosterone in amyloid processing. In fact, testosterone was shown to alter APP processing and enhance expression of neprilysin (Verdile et al. 2014).

Similarly, in 3xTgAD mice, GDX males presented an increased $A \beta$ accumulation in the brain, coupled with impaired cognitive performances compared to sham operated mice (Rosario et al. 2006). Treatment with androgens (testosterone or its metabolite dihydrotestosterone (DHT)) significantly attenuated the increase in AD pathology (Rosario et al. 2010; Rosario et al. 2006). Of note, testosterone can follow two enzymatic pathways: it can be converted to DHT by the enzyme $5 \alpha$-reductase or to estradiol by the enzyme aromatase. Thus, a treatment of GDX triple transgenic males with estradiol was also able to 
reduce $A \beta$ burden in the hippocampus, suggesting that both androgens and estrogens can influence APP processing and/or A $\beta$ degradation (Pike et al. 2009; Rosario et al. 2010). In an elegant study, Carroll and colleagues (Carroll et al. 2010a) investigated the influence of neonatal sex hormone exposure of male and female 3xTgAD mice on brain $A \beta$ accumulation. They found that demasculinized males exhibited a significant increase in cerebral $A \beta$ load compared to normal males, while in defeminized female mice $A \beta$ accumulation was comparable to that of males in some brain regions.

Together, these findings suggest that, with regards to $A \beta$ pathology, testosterone may have an upstream action compared to estradiol because it can influence $A \beta$ synthesis and degradation by following the androgenic and the estrogenic pathway (Fig. 3).

\subsection{Effects on tau pathology:}

NFTs, composed by the aggregation of abnormally hyperphosphorylated tau protein, are the second histopathological hallmark observed in AD brain. The influence of sex steroid hormones on tau phosphorylation was also a matter of investigation.

In vitro evidence showed that estradiol reduced the activity of protein kinase $A$, an enzyme involved in tau phosphorylation, in a model of human embryonic kidney cells (HEK293 cells) overexpressing tau (Liu et al. 2008). Similar results were obtained in primary rat cortical neurons and SH-SY5Y neuroblastoma cells where estradiol rescued aberrant tau (Alvarez-de-la-Rosa et al. 2005).

In 3xTgAD female mice, estradiol and progesterone (using both continuous and cyclic treatment paradigms) lowered tau hyperphosphorylation (Carroll et al. 2007; Carroll et al. 2010b). Possible mechanisms involved might be a reduction of glycogen synthase kinase $3 \beta$

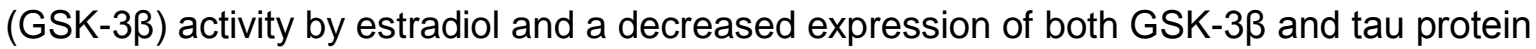
induced by progesterone (reviewed in (Pike et al. 2009)). Of note, GSK-3 $\beta$ is one of the main enzymes involved in tau hyperphosphorylation (Wang et al. 2013). 
Regarding the male hormones, testosterone was shown to prevent tau hyperphosphorylation in a model of heat shock-induced phosphorylation through inhibition of GSK signaling (Papasozomenos 1997). Rosario and colleagues (Rosario et al. 2010) demonstrated a less abnormal tau accumulation in GDX 3xTgAD males treated with testosterone. Interestingly, in these animals a treatment with estradiol, but not DHT, presented comparable effects to those of testosterone (Rosario et al. 2010; Rosario et al. 2006). These findings suggested that testosterone may exert effects on tau hyperphosphorylation via its aromatization in estradiol and by acting on estrogen receptors. In this model, androgens decreased $A \beta$ load with a higher efficacy compared to estradiol suggesting rather an androgen receptor-dependent mechanism.

Taken together, these findings demonstrated that steroid sex hormones are able to modulate both $A \beta$ and tau pathology (Fig. 3). Besides, a treatment with androgens was associated more with lesser $A \beta$ burden rather than a reduction in abnormal tau protein. Since testosterone can act via both estrogen and androgen receptors to modulate $A \beta$ generation/degradation, this might explain why females have more $A \beta$ deposits compared to males, due to their lower testosterone levels in general.

\section{New insights on protective effects of sex hormones against $A \beta$ and tau-related mitochondrial dysfunction}

Whether sex steroid hormones are able to rescue or alleviate AD-related mitochondrial deficits was the concern of several publications almost all focusing on estradiol.

Indeed, a growing body of evidence reports that estradiol can regulate bioenergetics and redox homeostasis (reviewed in (Grimm et al. 2012)). More specifically, estradiol was shown to: i) increase glucose metabolism by regulating the expression of genes involved in glucose transport, glycolysis and tricarboxylic acid cycle (TCA cycle); ii) up-regulate genes encoding for components of the mitochondrial electron transport chain (ETC) including 
complex I (CI), cytochrome c oxidase (complex IV), and the F1 subunit of ATP synthase; iii) up-regulate anti-oxidant defenses, such as MnSOD and GHS levels; iv) modulate the redox state of cells by acting on several signalling pathways, such as MAPK (mitogen activated protein kinase), G protein regulated signalling, NFKB, c-fos, CREB, phosphatidylinositol-3kinase, PKC and $\mathrm{Ca}^{2+}$ influx. On the basis of this complex mode of action, estradiol seems to be able to decrease oxidative stress markers, including lipid peroxidation, protein oxidation and DNA damage, and can also regulate mitochondrial respiratory function.

In OVX female rats, a 24 hour treatment with estradiol or progesterone increased OXPHOS capacity in isolated mitochondria, at least in part by enhancing COX activity as well as expression (Irwin et al. 2008). Interestingly, these effects were suppressed by a cotreatment with estradiol and progesterone, again suggesting a competitive mode of action between both steroids.

Surprisingly, the role of androgens in mitochondrial function has received only little attention until now compared to the estrogens. One study showed that testosterone increased the mitochondrial membrane potential in vitro (Holmes et al. 2013). Since both nuclear and mitochondrial DNA contain hormone responsive elements (estrogen and androgen receptors), it has been proposed that sex hormones can regulate bioenergetics by inducing the expression of genes involved in OXPHOS, but the exact mechanisms remain unclear (Vasconsuelo et al. 2013).

In line with these findings, we recently demonstrated that the steroid sex hormones estrogens (estradiol and estrone), progesterone and testosterone were able to improve bioenergetic activity in SH-SY5Y neuroblastoma cells and in primary neuronal culture by increasing ATP levels, MMP and basal mitochondrial respiration (Grimm et al. 2014). In parallel, they modulated redox homeostasis by increasing antioxidant activity, probably as a compensatory mechanism to a slight enhancement of ROS. These effects were mediated, at least in part, by steroid receptor activation. Thus, our results demonstrated that the ability to 
boost mitochondria was not unique to estradiol and that sex steroids represent attractive tools to counteract mitochondrial deficits in AD.

To continue in this direction, we evaluated the impact of sex steroids on mitochondrial dysfunction in cellular models of $A D$ overexpressing either APP/A $\beta$ or tau protein bearing the P301L mutation (Grimm et al. 2016a). We showed that the bioenergetic deficits induced by either $A \beta$ or abnormally hyperphosphorylated tau protein can be alleviated by a treatment with sex hormones. More specifically, testosterone represented the lead steroid acting against mitochondrial deficits induced by $A \beta$, while progesterone and estrogens were more efficient against AD-related tauopathies. These data are in line with previous studies showing that testosterone was more efficient in reducing $A \beta$ load than abnormal tau protein, in contrast to estradiol and progesterone that can modulate both $A \beta$ and tau pathology (Rosario et al. 2010; Rosario et al. 2006). Together, our findings lend further evidence to the neuroprotective effects of sex steroids in AD pathology and indicate that these molecules represent promising tools able to increase mitochondrial bioenergetics in pathological conditions (Fig. 3). Our results may open new avenues for the development of genderspecific therapeutic approaches in AD.

To our knowledge, no studies investigating the effects of progesterone or testosterone on AD-induced mitochondrial dysfunction were performed in vivo, but the effect of estradiol has been well characterized.

Indeed, in triple transgenic AD mice, it was shown that OVX females presented stronger bioenergetic deficits, namely a decline in mitochondrial respiration accompanied by an increase in oxidative stress when compared to intact animals (Yao et al. 2012). In addition, $A \beta$ load was more important in mitochondria and in the hippocampus of transgenic OVX animals. Of note, in this model, mitochondrial energy deficiency was detected even before the onset of $A D$ pathology (Yao et al. 2009). Similar observations were also made in a study investigating the impact of natural reproductive senescence on mitochondrial bioenergetics in 3xTgAD females (Yao et al. 2010). In this model, OXPHOS, PDH and COX 
activity was even more decreased in transgenic $A D$ animals compared to non-transgenic littermates especially at an age between 9 and 12 months, a vulnerable age which corresponds to the onset of reproductive senescence in mice. At these ages, a shift to fatty acid / ketone metabolism in the brain was observed in female rodents, probably as a compensatory mechanism to the decrease of mitochondrial activity (Yao et al. 2010; Yin et al. 2015).

In 3xTgAD mice, estrogen replacement in OVX animals was able to alleviate / normalize bioenergetic and redox parameters as well as to reduce $A \beta$ burden (Yao et al. 2012), again highlighting the protective effects of this sex hormone against AD pathology.

Finally, to gain insights into the pathological mechanisms of $A \beta$ upon mitochondria, recent studies focused on a mitochondrial enzyme called $A \beta$-binding alcohol dehydrogenase (ABAD). This enzyme is overexpressed in the brain of AD patients as well as OVX $3 \times \operatorname{Tg} A D$ mice, and seems to play a role in mitochondrial estradiol metabolism (Yan et al. 1997; Yao et al. 2012). Based on findings showing that inhibition of $A \beta-A B A D$ interaction by a decoy peptide can restore mitochondrial deficits in APP mice, we recently investigated the role of a novel small $A B A D$-specific compound inhibitor (AG18051) on $A \beta$-induced mitochondrial toxicity and estradiol metabolism in SH-SY5Y cells (Lim et al. 2011). We found that AG18051 partially blocked $A \beta-A B A D$ interaction and prevented the $A \beta$-induced down-regulation of ABAD activity by normalizing estradiol levels. Furthermore, $A G 18051$ reduced $A \beta$-induced impairment of mitochondrial respiration, oxidative stress and cell death. Thus, our results emphasized the inhibition of ABAD by compounds such as AG18051 as a promising therapeutic strategy for the prevention and/or treatment of $A D$ and suggest that the endogenous modulation of mitochondrial estradiol metabolism is important for mitochondrial activity (Grimm et al. 2012).

\section{Hormone replacement therapy: successes and controversies}


Mounting evidence coming from cellular and animal models attests to neuroprotective effects of steroid hormones in $A D$, but clinical trial data remain controversial. Hormone replacement therapy (HRT) involves the administration of synthetic estrogen and progesterone to replace a woman's depleting hormone level. However, HRT has been linked to various risks and the debate regarding risk-benefit ratio continues.

At first, promising studies showed that the use of estrogen in postmenopausal women reduced the risk of AD or delayed the onset of the disease (Fillit 2002; Paganini-Hill and Henderson 1996; Tang et al. 1996). But, beneficial effects of HRT have been challenged by results from the "Woman's health initiative memory study" (WHIMS), which included 4,532 postmenopausal women aged over 68 years and showed negative effects of long-term HRT (Anderson et al. 2004; Rossouw et al. 2002). Data revealed at a two-fold increase in dementia after 4.2 years of hormonal treatment (p.o. treatment with premaxin plus medroxyprogesterone) as well as increased risks for breast cancer, pulmonary embolism and stroke. Criticism of this study concerns mainly the following points:

i) One criticism is directed towards the synthetic nature of the hormones used in the WHIMS trial, conjugated equine estrogen (CCE) and medroxyprogesterone, instead of using natural hormones. Indeed, protective effects of estradiol can be antagonized by a co-treatment with medroxyprogesterone, which showed no protective action against seizure, cortical contusion, ischaemia and diabetic neuropathy unlike estradiol, progesterone and its metabolite allopregnanolone (Frye and Walf 2008; Goodman et al. 1996; Nilsen and Brinton 2002). Of note, since continuous progesterone administration was also shown to antagonize estradiol effects in vivo (Carroll et al. 2007; Carroll et al. 2010b), the use of a cyclic treatment regime might also be considered in women in the future.

ii) The second criticism to the WHIMS focused on the age of the participants that were treated almost 10 years after the menopause. To highlight the importance of the period of time in which the treatment has to be initiated, Rocca and colleagues recently published an update of clinical outcomes of HRT (Rocca et al. 2014). They showed that when HRT starts 
shortly after the menopause, women exhibited a reduced risk of cognitive decline or dementia. However, when the initiation of HRT is delayed, as in the WHIMS study, the risk of cognitive deterioration is increased. This "critical window hypothesis" is substantiated by animal studies testing the effects of early versus late ERT after OVX (Henderson and Brinton 2010; Lopez-Grueso et al. 2014). Consistent with observations performed in humans, estrogen was efficient in reducing metabolic as well as redox impairments only when animals received treatment immediately after OVX and not few months later (Lopez-Grueso et al. 2014). One explanation was that long term estrogen depletion induced a change in the expression of estrogen receptor which decreased the sensitivity to this hormone with time (Lopez-Grueso et al. 2014; Scott et al. 2012).

To go further, Brinton proposed a complementary hypothesis called "the healthy cell bias of estrogen action" (Brinton 2008). This theory predicts that estrogen can exert a beneficial and protective effect only before exposure to neurodegenerative insults, such as oxidative stress, $A \beta$ toxicity and mitochondria impairments. In contrast, when bioenergetic and redox deregulation are already initiated, the presence of estrogen can exacerbate neurological demise. Thus the "critical window of treatment opportunity" may be placed at the perimenopausal age, when sex hormone levels are decreasing and brain bioenergetic and redox state start to change (Fig. 1).

Since men do not experience a decrease of sex hormones as dramatic as women, their "critical window" of treatment is more tricky to delimit (Fig. 1). One can imagine that the slow decrease of testosterone with increasing age might be coupled with slower and late onset in the changes of the bioenergetic status, and an androgen replacement therapy (ART) would start later (between 60-65 years old) compared to ERT in female (between 50-55 years old).

In comparison to the wide data base available about clinical trial of HRT in women, only few studies focused on the effects ART on the risk to develop AD in older men. Some data showed that ART improved overall quality of life in AD patients without significant effects on cognition (Lu et al. 2006). More recently, a double-blind randomized clinical trial 
was performed on 44 older men (aged $61 \pm 7.7$ years) with subjective memory complains to assess ART (here testosterone) physiological effects (Asih et al. 2015). Preliminary results showed that testosterone was well-tolerated, without notable side effects, but longitudinal proof-of-concept studies are now needed to assess whether ART is efficient in reducing AD risks in men.

Taken together, these findings propose that, despite the failure of the WHIMS trial, the identification of an optimal window of treatment opportunity as well as safe doses and suitable formulation may hopefully allow a breakthrough in the use of HRT as AD preventing strategy in both women and men. Besides, new treatment alternatives, using specific estrogen/androgen receptor modulator (SERM/SARM) are currently under investigation for the treatment of $A D$. This notably includes the use of phytoestrogen acting selectively on estrogen receptor $\beta$, that is devoid of feminizing activity in the periphery unlike estrogen receptor $\alpha$, limiting unwanted side effects (Zhao et al. 2013).

\section{Conclusion}

In this review, we showed that aging and reproductive senescence is marked by a raise in brain oxidative stress coupled with bioenergetic impairments. The latters are both important features of early disease stages in AD pathogenesis, indicating that the decrease of sex hormones, at the menopause in women and andropause in men, may increase the risk to develop AD due to the loss of their neuroprotective effects. Indeed, both male and female sex hormones were shown to prevent, or at least slow down, the appearance of $A \beta$ plaques and NFTs in animal models of the disease.

Gender differences observed in human brain are consistent with that in animal models showing a higher vulnerability of females to oxidative injury and $A \beta$ pathology. Of note, differences in tau pathology between females and males are more equivocal and deserve more investigational attention in the future. Based on the "AD mitochondrial cascade hypothesis" (Swerdlow and Khan 2004), one can suggest that redox imbalance and 
bioenergetic impairment may be more prompt to pass a pathological threshold in women who experience a drastic drop of sex hormones within a short period of time at the menopause and, consequently, are less prepared to face oxidative injury and $A \beta$ accumulation compared to men (Fig. 4). Besides, the male sex hormone, testosterone, seems to be more powerful in counteracting $A \beta$ pathology compared to the female sex hormones (Barron and Pike 2012; Pike et al. 2009), since it can act via two pathways: i) testosterone can be converted in its androgenic metabolite DHT which acts via its androgen receptors, and/or ii) it is aromatized into estradiol acting via estrogen receptors. Thus, both pathways may influence $A \beta$ generation/degradation, leading to a decrease in $A \beta$ load.

Moreover, new findings lend further evidence to the neuroprotective effects of sex hormones against $A \beta$ and tau-induced mitochondrial dysfunction, indicating that these molecules represent promising tools able to increase mitochondrial bioenergetics in pathological conditions (Grimm et al. 2016a; Grimm et al. 2014).

Taken together, a substantial body of evidence illustrates the complex relationship between $A D$, mitochondria and sex hormones; anyhow many questions remain to be answered: what are the underlying molecular mechanisms? What is the best HRT paradigm? What is the optimal window of treatment opportunity in women or in men? Potentially, mitochondria may represent a key piece of the puzzle aiming for the understanding of the molecular basis of sex differences in AD which may open new avenues for the development of gender-specific therapeutic approaches. 


\section{Acknowledgments}

This work was supported by grants from Synapsis Foundation, Novartis Foundation for

Biomedical Research Basel and the Swiss National Science Foundation (\#31003A_149728) to $A E$. 


\section{References}

Adam-Vizi V, Chinopoulos C (2006) Bioenergetics and the formation of mitochondrial reactive oxygen species Trends in pharmacological sciences 27:639-645 doi:10.1016/j.tips.2006.10.005

Alvarez-de-la-Rosa M, Silva I, Nilsen J, Perez MM, Garcia-Segura LM, Avila J, Naftolin F (2005) Estradiol prevents neural tau hyperphosphorylation characteristic of Alzheimer's disease Ann N Y Acad Sci 1052:210-224 doi:10.1196/annals.1347.016

Amtul Z, Wang L, Westaway D, Rozmahel RF (2010) Neuroprotective mechanism conferred by 17beta-estradiol on the biochemical basis of Alzheimer's disease Neuroscience 169:781-786 doi:S0306-4522(10)00751-7 [pii] 10.1016/j.neuroscience.2010.05.031

Anderson GL et al. (2004) Effects of conjugated equine estrogen in postmenopausal women with hysterectomy: the Women's Health Initiative randomized controlled trial Journal of the American Medical Association 291:1701-1712 doi:10.1001/jama.291.14.1701 291/14/1701 [pii]

Asih PR et al. (2015) Testosterone replacement therapy in older male subjective memory complainers: double-blind randomized crossover placebo-controlled clinical trial of physiological assessment and safety CNS Neurol Disord Drug Targets 14:576-586

Balaban RS, Nemoto S, Finkel T (2005) Mitochondria, oxidants, and aging Cell 120:483-495 doi:10.1016/j.cell.2005.02.001

Barnes LL, Wilson RS, Bienias JL, Schneider JA, Evans DA, Bennett DA (2005) Sex differences in the clinical manifestations of Alzheimer disease pathology Archives of general psychiatry 62:685-691 doi:10.1001/archpsyc.62.6.685

Barron AM, Pike CJ (2012) Sex hormones, aging, and Alzheimer's disease Front Biosci (Elite Ed) 4:976-997

Bellanti F, Matteo M, Rollo T, De Rosario F, Greco P, Vendemiale G, Serviddio G (2013) Sex hormones modulate circulating antioxidant enzymes: impact of estrogen therapy Redox biology 1:340-346 doi:10.1016/j.redox.2013.05.003

Borras C, Sastre J, Garcia-Sala D, Lloret A, Pallardo FV, Vina J (2003) Mitochondria from females exhibit higher antioxidant gene expression and lower oxidative damage than males Free Radical Biology and Medicine 34:546-552 doi:S0891584902013564 [pii]

Brinton RD (2008) The healthy cell bias of estrogen action: mitochondrial bioenergetics and neurological implications Trends in neurosciences 31:529-537 doi:10.1016/j.tins.2008.07.003

Brookmeyer R, Johnson E, Ziegler-Graham K, Arrighi HM (2007) Forecasting the global burden of Alzheimer's disease Alzheimer's \& dementia : the journal of the Alzheimer's Association 3:186-191 doi:10.1016/j.jalz.2007.04.381 
Brunden KR, Trojanowski JQ, Lee VM (2009) Advances in tau-focused drug discovery for Alzheimer's disease and related tauopathies Nature reviews Drug discovery 8:783793 doi: $10.1038 /$ nrd2959

Callahan MJ, Lipinski WJ, Bian F, Durham RA, Pack A, Walker LC (2001) Augmented senile plaque load in aged female beta-amyloid precursor protein-transgenic mice The American journal of pathology 158:1173-1177

Campello S, Scorrano L (2010) Mitochondrial shape changes: orchestrating cell pathophysiology EMBO Rep 11:678-684 doi:10.1038/embor.2010.115

Carroll JC, Rosario ER, Chang L, Stanczyk FZ, Oddo S, LaFerla FM, Pike CJ (2007) Progesterone and estrogen regulate Alzheimer-like neuropathology in female 3xTgAD mice Journal of Neuroscience 27:13357-13365 doi:27/48/13357 [pii] 10.1523/JNEUROSCI.2718-07.2007

Carroll JC, Rosario ER, Kreimer S, Villamagna A, Gentzschein E, Stanczyk FZ, Pike CJ (2010a) Sex differences in beta-amyloid accumulation in 3xTg-AD mice: role of neonatal sex steroid hormone exposure Brain Res 1366:233-245 doi:10.1016/j.brainres.2010.10.009

Carroll JC, Rosario ER, Villamagna A, Pike CJ (2010b) Continuous and cyclic progesterone differentially interact with estradiol in the regulation of Alzheimer-like pathology in female 3xTransgenic-Alzheimer's disease mice Endocrinology 151:2713-2722 doi:10.1210/en.2009-1487

Caruso D et al. (2013) Age-related changes in neuroactive steroid levels in 3xTg-AD mice Neurobiol Aging 34:1080-1089 doi:10.1016/j.neurobiolaging.2012.10.007 S01974580(12)00497-6 [pii]

Corder EH, Ghebremedhin E, Taylor MG, Thal DR, Ohm TG, Braak H (2004) The biphasic relationship between regional brain senile plaque and neurofibrillary tangle distributions: modification by age, sex, and APOE polymorphism Ann N Y Acad Sci 1019:24-28 doi:10.1196/annals.1297.005

Corpechot C, Robel P, Axelson M, Sjovall J, Baulieu EE (1981) Characterization and measurement of dehydroepiandrosterone sulfate in rat brain Proc Natl Acad Sci U S A 78:4704-4707

Cunningham RL, Singh M, O'Bryant SE, Hall JR, Barber RC (2014) Oxidative stress, testosterone, and cognition among Caucasian and Mexican-American men with and without Alzheimer's disease J Alzheimers Dis 40:563-573 doi:10.3233/JAD-131994

David DC et al. (2005) Proteomic and functional analyses reveal a mitochondrial dysfunction in P301L tau transgenic mice J Biol Chem 280:23802-23814 doi:M500356200 [pii] 10.1074/jbc.M500356200 
Devi L, Alldred MJ, Ginsberg SD, Ohno M (2010) Sex- and brain region-specific acceleration of beta-amyloidogenesis following behavioral stress in a mouse model of Alzheimer's disease Molecular brain 3:34 doi:10.1186/1756-6606-3-34

DuBoff B, Feany M, Gotz J (2013) Why size matters - balancing mitochondrial dynamics in Alzheimer's disease Trends in neurosciences 36:325-335 doi:10.1016/j.tins.2013.03.002

DuBoff B, Gotz J, Feany MB (2012) Tau promotes neurodegeneration via DRP1 mislocalization in vivo Neuron 75:618-632 doi:10.1016/j.neuron.2012.06.026 S08966273(12)00588-0 [pii]

Dumont $\mathrm{M}$ et al. (2011) Behavioral deficit, oxidative stress, and mitochondrial dysfunction precede tau pathology in P301S transgenic mice FASEB J 25:4063-4072 doi:10.1096/fj.11-186650

Eckert A et al. (2008) Oligomeric and fibrillar species of beta-amyloid (A beta 42) both impair mitochondrial function in P301L tau transgenic mice $\mathrm{J}$ Mol Med (Berl) 86:1255-1267 doi:10.1007/s00109-008-0391-6

Eckert A, Nisbet R, Grimm A, Gotz J (2014) March separate, strike together--role of phosphorylated TAU in mitochondrial dysfunction in Alzheimer's disease Biochim Biophys Acta 1842:1258-1266 doi:10.1016/j.bbadis.2013.08.013

Eckert A, Schmitt K, Götz J (2011) Mitochondrial dysfunction - the beginning of the end in Alzheimer's disease? Separate and synergistic modes of tau and amyloid-beta toxicity Alzheimers Res Ther 3:15 doi:10.1186/alzrt74

Eckert A, Schulz KL, Rhein V, Gotz J (2010) Convergence of amyloid-beta and tau pathologies on mitochondria in vivo Mol Neurobiol 41:107-114 doi:10.1007/s12035010-8109-5

Feldman HA et al. (2002) Age trends in the level of serum testosterone and other hormones in middle-aged men: longitudinal results from the Massachusetts male aging study $\mathrm{J}$ Clin Endocrinol Metab 87:589-598 doi:10.1210/jcem.87.2.8201

Fillit HM (2002) The role of hormone replacement therapy in the prevention of Alzheimer disease Arch Intern Med 162:1934-1942

Frye CA, Walf AA (2008) Effects of progesterone administration and APPswe+PSEN1Deltae9 mutation for cognitive performance of mid-aged mice Neurobiol Learn Mem 89:17-26 doi:S1074-7427(07)00161-X [pii] 10.1016/j.nlm.2007.09.008

Gallagher JJ, Minogue AM, Lynch MA (2013) Impaired performance of female APP/PS1 mice in the Morris water maze is coupled with increased Abeta accumulation and microglial activation Neurodegener Dis 11:33-41 doi:10.1159/000337458 
Goedert M, Jakes R (2005) Mutations causing neurodegenerative tauopathies Biochim Biophys Acta 1739:240-250 doi:10.1016/j.bbadis.2004.08.007

Goodman Y, Bruce AJ, Cheng B, Mattson MP (1996) Estrogens attenuate and corticosterone exacerbates excitotoxicity, oxidative injury, and amyloid beta-peptide toxicity in hippocampal neurons J Neurochem 66:1836-1844

Götz J, Chen F, van Dorpe J, Nitsch RM (2001) Formation of neurofibrillary tangles in P301I tau transgenic mice induced by Abeta 42 fibrils Science 293:1491-1495 doi:10.1126/science.1062097 293/5534/1491 [pii]

Götz J, Ittner LM (2008) Animal models of Alzheimer's disease and frontotemporal dementia Nat Rev Neurosci 9:532-544 doi:nrn2420 [pii] 10.1038/nrn2420

Grimm A, Biliouris EE, Lang UE, Götz J, Mensah-Nyagan AG, Eckert A (2016a) Sex hormone-related neurosteroids differentially rescue bioenergetic deficits induced by amyloid-beta or hyperphosphorylated tau protein Cellular and molecular life sciences : CMLS 73:201-215 doi:10.1007/s00018-015-1988-x

Grimm A, Friedland K, Eckert A (2016b) Mitochondrial dysfunction: the missing link between aging and sporadic Alzheimer's disease Biogerontology 17:281-296 doi:10.1007/s10522-015-9618-4

Grimm A, Lim YA, Mensah-Nyagan AG, Götz J, Eckert A (2012) Alzheimer's disease, oestrogen and mitochondria: an ambiguous relationship Mol Neurobiol 46:151-160 doi:10.1007/s12035-012-8281-x

Grimm A, Schmitt K, Lang UE, Mensah-Nyagan AG, Eckert A (2014) Improvement of neuronal bioenergetics by neurosteroids: Implications for age-related neurodegenerative disorders Biochim Biophys Acta doi:10.1016/j.bbadis.2014.09.013

Grueninger F et al. (2010) Phosphorylation of Tau at S422 is enhanced by Abeta in TauPS2APP triple transgenic mice Neurobiol Dis 37:294-306 doi:S09699961(09)00245-9 [pii] 10.1016/j.nbd.2009.09.004

Guglielmotto M et al. (2009) The up-regulation of BACE1 mediated by hypoxia and ischemic injury: role of oxidative stress and HIF1alpha J Neurochem 108:1045-1056 doi:10.1111/j.1471-4159.2008.05858.x

Harman D (1956) Aging: a theory based on free radical and radiation chemistry Journal of gerontology 11:298-300

Harper ME, Bevilacqua L, Hagopian K, Weindruch R, Ramsey JJ (2004) Ageing, oxidative stress, and mitochondrial uncoupling Acta physiologica Scandinavica 182:321-331 doi:10.1111/j.1365-201X.2004.01370.x

Henderson VW, Brinton RD (2010) Menopause and mitochondria: windows into estrogen effects on Alzheimer's disease risk and therapy Progress in brain research 182:77-96 doi:10.1016/S0079-6123(10)82003-5 
Hirata-Fukae C et al. (2008) Females exhibit more extensive amyloid, but not tau, pathology in an Alzheimer transgenic model Brain Res 1216:92-103 doi:10.1016/j.brainres.2008.03.079

Holmes S, Abbassi B, Su C, Singh M, Cunningham RL (2013) Oxidative stress defines the neuroprotective or neurotoxic properties of androgens in immortalized female rat dopaminergic neuronal cells Endocrinology 154:4281-4292 doi:10.1210/en.20131242

Irwin RW, Yao J, Hamilton RT, Cadenas E, Brinton RD, Nilsen J (2008) Progesterone and estrogen regulate oxidative metabolism in brain mitochondria Endocrinology 149:3167-3175 doi:en.2007-1227 [pii] 10.1210/en.2007-1227

Ittner LM, Gotz J (2011) Amyloid-beta and tau--a toxic pas de deux in Alzheimer's disease Nat Rev Neurosci 12:65-72 doi:10.1038/nrn2967

Jayaraman A et al. (2012) 17beta-estradiol and progesterone regulate expression of betaamyloid clearance factors in primary neuron cultures and female rat brain Endocrinology 153:5467-5479 doi:10.1210/en.2012-1464

Jezek P, Hlavata L (2005) Mitochondria in homeostasis of reactive oxygen species in cell, tissues, and organism The international journal of biochemistry \& cell biology 37:2478-2503 doi:10.1016/j.biocel.2005.05.013

Kalous M, Drahota Z (1996) The role of mitochondria in aging Physiological research / Academia Scientiarum Bohemoslovaca 45:351-359

Keil $U$ et al. (2004) Amyloid beta-induced changes in nitric oxide production and mitochondrial activity lead to apoptosis J Biol Chem 279:50310-50320 doi:10.1074/jbc.M405600200

King DL, Arendash GW, Crawford F, Sterk T, Menendez J, Mullan MJ (1999) Progressive and gender-dependent cognitive impairment in the APP(SW) transgenic mouse model for Alzheimer's disease Behav Brain Res 103:145-162

Kremer A, Maurin H, Demedts D, Devijver H, Borghgraef P, Van Leuven F (2011) Early improved and late defective cognition is reflected by dendritic spines in Tau.P301L mice J Neurosci 31:18036-18047 doi:10.1523/JNEUROSCI.4859-11.2011

LaFerla FM, Green KN, Oddo S (2007) Intracellular amyloid-beta in Alzheimer's disease Nat Rev Neurosci 8:499-509 doi:10.1038/nrn2168

Lee HC, Wei YH (1997) Mutation and oxidative damage of mitochondrial DNA and defective turnover of mitochondria in human aging Journal of the Formosan Medical Association = Taiwan yi zhi 96:770-778

Leuner K, Muller WE, Reichert AS (2012a) From mitochondrial dysfunction to amyloid beta formation: novel insights into the pathogenesis of Alzheimer's disease Mol Neurobiol 46:186-193 doi:10.1007/s12035-012-8307-4 
Leuner K et al. (2012b) Mitochondrion-derived reactive oxygen species lead to enhanced amyloid beta formation Antioxid Redox Signal 16:1421-1433

doi:10.1089/ars.2011.4173

Lewis $\mathrm{J}$ et al. (2001) Enhanced neurofibrillary degeneration in transgenic mice expressing mutant tau and APP Science 293:1487-1491 doi:10.1126/science.1058189 293/5534/1487 [pii]

Lim YA et al. (2011) Inhibition of the Mitochondrial Enzyme ABAD Restores the Amyloidbeta-Mediated Deregulation of Estradiol PLoS One 6:e28887 doi:10.1371/journal.pone.0028887 PONE-D-11-14664 [pii]

Liu XA, Zhu LQ, Zhang Q, Shi HR, Wang SH, Wang Q, Wang JZ (2008) Estradiol attenuates tau hyperphosphorylation induced by upregulation of protein kinase-A Neurochem Res 33:1811-1820 doi:10.1007/s11064-008-9638-4

Lopez-Grueso R et al. (2014) Early, but not late onset estrogen replacement therapy prevents oxidative stress and metabolic alterations caused by ovariectomy Antioxid Redox Signal 20:236-246 doi:10.1089/ars.2012.5112

Lu PH et al. (2006) Effects of testosterone on cognition and mood in male patients with mild Alzheimer disease and healthy elderly men Archives of neurology 63:177-185 doi:10.1001/archneur.63.2.nct50002

Mandal PK, Tripathi M, Sugunan S (2012) Brain oxidative stress: detection and mapping of anti-oxidant marker 'Glutathione' in different brain regions of healthy male/female, $\mathrm{MCl}$ and Alzheimer patients using non-invasive magnetic resonance spectroscopy Biochemical and biophysical research communications 417:43-48 doi:10.1016/j.bbrc.2011.11.047

Melcangi RC, Giatti S, Garcia-Segura LM (2015) Levels and actions of neuroactive steroids in the nervous system under physiological and pathological conditions: Sex-specific features Neuroscience and biobehavioral reviews doi:10.1016/j.neubiorev.2015.09.023

Meydan S et al. (2010) Effects of testosterone on orchiectomy-induced oxidative damage in the rat hippocampus Journal of chemical neuroanatomy 40:281-285 doi:10.1016/j.jchemneu.2010.07.006

Mielke MM, Vemuri P, Rocca WA (2014) Clinical epidemiology of Alzheimer's disease: assessing sex and gender differences Clinical epidemiology 6:37-48 doi:10.2147/CLEP.S37929

Nilsen J, Brinton RD (2002) Impact of progestins on estrogen-induced neuroprotection: synergy by progesterone and 19-norprogesterone and antagonism by medroxyprogesterone acetate Endocrinology 143:205-212 
Overk CR, Lu PY, Wang YT, Choi J, Shaw JW, Thatcher GR, Mufson EJ (2012) Effects of aromatase inhibition versus gonadectomy on hippocampal complex amyloid pathology in triple transgenic mice Neurobiol Dis 45:479-487 doi:10.1016/j.nbd.2011.08.035

Overk CR, Perez SE, Ma C, Taves MD, Soma KK, Mufson EJ (2013) Sex steroid levels and AD-like pathology in 3xTgAD mice Journal of neuroendocrinology 25:131-144 doi:10.1111/j.1365-2826.2012.02374.x

Paganini-Hill A, Henderson VW (1996) Estrogen replacement therapy and risk of Alzheimer disease Archives of Internal Medicine 156:2213-2217

Papasozomenos SC (1997) The heat shock-induced hyperphosphorylation of tau is estrogen-independent and prevented by androgens: implications for Alzheimer disease Proc Natl Acad Sci U S A 94:6612-6617

Perry G, Nunomura A, Hirai K, Takeda A, Aliev G, Smith MA (2000) Oxidative damage in Alzheimer's disease: the metabolic dimension Int J Dev Neurosci 18:417-421

Peskind ER et al. (2014) Influence of lifestyle modifications on age-related free radical injury to brain JAMA neurology 71:1150-1154 doi:10.1001/jamaneurol.2014.1428

Pike CJ, Carroll JC, Rosario ER, Barron AM (2009) Protective actions of sex steroid hormones in Alzheimer's disease Front Neuroendocrinol 30:239-258 doi:S00913022(09)00024-7 [pii] 10.1016/j.yfrne.2009.04.015

Prince M, Bryce R, Albanese E, Wimo A, Ribeiro W, Ferri CP (2013) The global prevalence of dementia: a systematic review and metaanalysis Alzheimer's \& dementia : the journal of the Alzheimer's Association 9:63-75 e62 doi:10.1016/j.jalz.2012.11.007

Reitz C, Mayeux R (2014) Alzheimer disease: epidemiology, diagnostic criteria, risk factors and biomarkers Biochemical pharmacology 88:640-651 doi:10.1016/j.bcp.2013.12.024

Rekkas PV et al. (2014) Greater monoamine oxidase a binding in perimenopausal age as measured with carbon 11-labeled harmine positron emission tomography JAMA psychiatry 71:873-879 doi:10.1001/jamapsychiatry.2014.250

Rhein V, Baysang G, Rao S, Meier F, Bonert A, Muller-Spahn F, Eckert A (2009a) Amyloidbeta leads to impaired cellular respiration, energy production and mitochondrial electron chain complex activities in human neuroblastoma cells Cell Mol Neurobiol 29:1063-1071 doi:10.1007/s10571-009-9398-y

Rhein V et al. (2009b) Amyloid-beta and tau synergistically impair the oxidative phosphorylation system in triple transgenic Alzheimer's disease mice Proc Natl Acad Sci U S A 106:20057-20062 doi:10.1073/pnas.0905529106 0905529106 [pii] 
Rocca WA, Grossardt BR, Shuster LT (2014) Oophorectomy, estrogen, and dementia: a 2014 update Molecular and cellular endocrinology 389:7-12 doi:10.1016/j.mce.2014.01.020

Rone MB, Fan J, Papadopoulos V (2009) Cholesterol transport in steroid biosynthesis: role of protein-protein interactions and implications in disease states Biochimica et Biophysica Acta 1791:646-658 doi:S1388-1981(09)00071-7 [pii] 10.1016/j.bbalip.2009.03.001

Rosario ER, Carroll J, Pike CJ (2010) Testosterone regulation of Alzheimer-like neuropathology in male 3xTg-AD mice involves both estrogen and androgen pathways Brain Res 1359:281-290 doi:10.1016/j.brainres.2010.08.068

Rosario ER, Carroll JC, Oddo S, LaFerla FM, Pike CJ (2006) Androgens regulate the development of neuropathology in a triple transgenic mouse model of Alzheimer's disease J Neurosci 26:13384-13389 doi:10.1523/JNEUROSCI.2514-06.2006

Rosario ER, Chang L, Head EH, Stanczyk FZ, Pike CJ (2011) Brain levels of sex steroid hormones in men and women during normal aging and in Alzheimer's disease Neurobiol Aging 32:604-613 doi:10.1016/j.neurobiolaging.2009.04.008

Rossouw JE et al. (2002) Risks and benefits of estrogen plus progestin in healthy postmenopausal women: principal results From the Women's Health Initiative randomized controlled trial Journal of the American Medical Association 288:321-333 doi:joc21036 [pii]

Schmitt K, Grimm A, Kazmierczak A, Strosznajder JB, Götz J, Eckert A (2012) Insights into mitochondrial dysfunction: aging, amyloid-beta, and tau-A deleterious trio Antioxid Redox Signal 16:1456-1466 doi:10.1089/ars.2011.4400

Schuessel K, Leutner S, Cairns NJ, Muller WE, Eckert A (2004) Impact of gender on upregulation of antioxidant defence mechanisms in Alzheimer's disease brain Journal of Neural Transmission 111:1167-1182 doi:10.1007/s00702-004-0156-5

Schulz KL et al. (2012) A new link to mitochondrial impairment in tauopathies Mol Neurobiol 46:205-216 doi:10.1007/s12035-012-8308-3

Scott E, Zhang QG, Wang R, Vadlamudi R, Brann D (2012) Estrogen neuroprotection and the critical period hypothesis Front Neuroendocrinol 33:85-104 doi:10.1016/j.yfrne.2011.10.001

Shulman RG, Rothman DL, Behar KL, Hyder F (2004) Energetic basis of brain activity: implications for neuroimaging Trends in neurosciences 27:489-495 doi:10.1016/j.tins.2004.06.005

Skup $M$ et al. (2011) Sex differences in grey matter atrophy patterns among $A D$ and $\mathrm{aMCI}$ patients: results from ADNI Neurolmage 56:890-906 doi:10.1016/j.neuroimage.2011.02.060 
Song L et al. (2015) Analysis of tau post-translational modifications in rTg4510 mice, a model of tau pathology Mol Neurodegener 10:14 doi:10.1186/s13024-015-0011-1

Stockburger C, Gold VA, Pallas T, Kolesova N, Miano D, Leuner K, Muller WE (2014) A cell model for the initial phase of sporadic Alzheimer's disease J Alzheimers Dis 42:395411 doi:10.3233/JAD-140381

Swerdlow RH, Burns JM, Khan SM (2014) The Alzheimer's disease mitochondrial cascade hypothesis: progress and perspectives Biochim Biophys Acta 1842:1219-1231 doi:10.1016/j.bbadis.2013.09.010

Swerdlow RH, Khan SM (2004) A "mitochondrial cascade hypothesis" for sporadic Alzheimer's disease Medical hypotheses 63:8-20 doi:10.1016/j.mehy.2003.12.045

Tamagno E et al. (2008) Oxidative stress activates a positive feedback between the gammaand beta-secretase cleavages of the beta-amyloid precursor protein $\mathrm{J}$ Neurochem 104:683-695 doi:10.1111/j.1471-4159.2007.05072.x

Tamagno $E$ et al. (2009) JNK and ERK1/2 pathways have a dual opposite effect on the expression of BACE1 Neurobiol Aging 30:1563-1573 doi:10.1016/j.neurobiolaging.2007.12.015

Tamagno E et al. (2005) Beta-site APP cleaving enzyme up-regulation induced by 4hydroxynonenal is mediated by stress-activated protein kinases pathways $\mathrm{J}$ Neurochem 92:628-636 doi:10.1111/j.1471-4159.2004.02895.x

Tang MX et al. (1996) Effect of oestrogen during menopause on risk and age at onset of Alzheimer's disease Lancet 348:429-432 doi:S0140-6736(96)03356-9 [pii] 10.1016/S0140-6736(96)03356-9

Trushina E et al. (2012) Defects in mitochondrial dynamics and metabolomic signatures of evolving energetic stress in mouse models of familial Alzheimer's disease PLoS One 7:e32737 doi:10.1371/journal.pone.0032737

Turrens JF (2003) Mitochondrial formation of reactive oxygen species The Journal of physiology 552:335-344 doi:10.1113/jphysiol.2003.049478

Van Dam D, De Deyn PP (2006) Drug discovery in dementia: the role of rodent models Nature reviews Drug discovery 5:956-970 doi:10.1038/nrd2075

Vasconsuelo A, Milanesi L, Boland R (2013) Actions of 17beta-estradiol and testosterone in the mitochondria and their implications in aging Ageing research reviews 12:907-917 doi:10.1016/j.arr.2013.09.001

Velarde MC (2014) Mitochondrial and sex steroid hormone crosstalk during aging Longevity \& healthspan 3:2 doi:10.1186/2046-2395-3-2

Verdile $\mathrm{G}$ et al. (2014) Associations between gonadotropins, testosterone and beta amyloid in men at risk of Alzheimer's disease Mol Psychiatry 19:69-75 doi:10.1038/mp.2012.147 
Vest RS, Pike CJ (2013) Gender, sex steroid hormones, and Alzheimer's disease Horm Behav 63:301-307 doi:10.1016/j.yhbeh.2012.04.006

Vina J, Borras C (2010) Women live longer than men: understanding molecular mechanisms offers opportunities to intervene by using estrogenic compounds Antioxid Redox Signal 13:269-278 doi:10.1089/ars.2009.2952

Vina $\mathrm{J}$ et al. (2007) Mitochondrial oxidant signalling in Alzheimer's disease $\mathrm{J}$ Alzheimers Dis $11: 175-181$

Wallace DC (2005) A mitochondrial paradigm of metabolic and degenerative diseases, aging, and cancer: a dawn for evolutionary medicine Annual review of genetics 39:359-407 doi:10.1146/annurev.genet.39.110304.095751

Wang JZ, Xia YY, Grundke-lqbal I, Iqbal K (2013) Abnormal hyperphosphorylation of tau: sites, regulation, and molecular mechanism of neurofibrillary degeneration $\mathrm{J}$ Alzheimers Dis 33 Suppl 1:S123-139 doi:10.3233/JAD-2012-129031

Wang X, Su B, Fujioka H, Zhu X (2008a) Dynamin-like protein 1 reduction underlies mitochondrial morphology and distribution abnormalities in fibroblasts from sporadic Alzheimer's disease patients The American journal of pathology 173:470-482 doi:10.2353/ajpath.2008.071208

Wang X, Su B, Lee HG, Li X, Perry G, Smith MA, Zhu X (2009) Impaired balance of mitochondrial fission and fusion in Alzheimer's disease $\mathrm{J}$ Neurosci 29:9090-9103 doi:10.1523/JNEUROSCI.1357-09.2009

Wang X et al. (2008b) Amyloid-beta overproduction causes abnormal mitochondrial dynamics via differential modulation of mitochondrial fission/fusion proteins Proc Natl Acad Sci U S A 105:19318-19323 doi:10.1073/pnas.0804871105

Yan SD et al. (1997) An intracellular protein that binds amyloid-beta peptide and mediates neurotoxicity in Alzheimer's disease Nature 389:689-695 doi:10.1038/39522

Yao J, Hamilton RT, Cadenas E, Brinton RD (2010) Decline in mitochondrial bioenergetics and shift to ketogenic profile in brain during reproductive senescence Biochim Biophys Acta 1800:1121-1126 doi:10.1016/j.bbagen.2010.06.002

Yao J, Irwin R, Chen S, Hamilton R, Cadenas E, Brinton RD (2012) Ovarian hormone loss induces bioenergetic deficits and mitochondrial beta-amyloid Neurobiol Aging 33:1507-1521 doi:10.1016/j.neurobiolaging.2011.03.001 S0197-4580(11)00058-3 [pii] Yao J, Irwin RW, Zhao L, Nilsen J, Hamilton RT, Brinton RD (2009) Mitochondrial bioenergetic deficit precedes Alzheimer's pathology in female mouse model of Alzheimer's disease Proc Natl Acad Sci U S A 106:14670-14675 doi:0903563106 [pii] 10.1073/pnas.0903563106 
Yin F, Boveris A, Cadenas E (2014) Mitochondrial energy metabolism and redox signaling in brain aging and neurodegeneration Antioxid Redox Signal 20:353-371 doi:10.1089/ars.2012.4774

Yin F et al. (2015) The perimenopausal aging transition in the female rat brain: decline in bioenergetic systems and synaptic plasticity Neurobiol Aging 36:2282-2295 doi:10.1016/j.neurobiolaging.2015.03.013

Yue M, Hanna A, Wilson J, Roder H, Janus C (2011) Sex difference in pathology and memory decline in rTg4510 mouse model of tauopathy Neurobiol Aging 32:590-603 doi:10.1016/j.neurobiolaging.2009.04.006

Yue $X$ et al. (2005) Brain estrogen deficiency accelerates Abeta plaque formation in an Alzheimer's disease animal model Proc Natl Acad Sci U S A 102:19198-19203 doi:10.1073/pnas.0505203102

Zhao L, Mao Z, Chen S, Schneider LS, Brinton RD (2013) Early intervention with an estrogen receptor beta-selective phytoestrogenic formulation prolongs survival, improves spatial recognition memory, and slows progression of amyloid pathology in a female mouse model of Alzheimer's disease J Alzheimers Dis 37:403-419 doi:10.3233/JAD122341 


\section{Figure captions:}

Fig. 1. Variation in sex steroid levels and theoretic redox state over life. Estrogen and testosterone levels rise at the puberty and are at their maximum during the reproductive life. Estrogen levels drop at the menopause in women when testosterone slowly decreases in men. Changes in the redox status (dotted lines) seem to be correlated with changes in sex hormones in both women and men. Hypothetical "critical windows" for hormone replacement therapy are indicated by squares.

Fig. 2. Gender-dependent hallmarks in transgenic AD mouse models. ( ${ }^{*}$ : with respect to gender differences: unpublished observations). $q$ : female; $\sigma^{\lambda}:$ male.

Fig. 3. Protective effects of sex hormones against $A D$ pathology. Testosterone $(T)$ can be converted in dihydrotestosterone (DHT) or estradiol (E2), both having $A \beta$ load-decreasing effects. In addition, estradiol and progesterone (P4) can decrease tau abnormal hyperphosphorylation and, thus, the formation of neurofibrillary tangles. $\leftrightarrow$ : P4 and E2 seem to have no synergistic but rather competitive effects. Testosterone, estradiol, and progesterone can rescue bioenergetic deficits observed in AD potentially by: i) inhibiting the toxic effects of $A \beta$ and abnormal tau on mitochondria; or ii) improving mitochondrial function under pathological conditions. P: phosphate.

Fig. 4. Potential sequence of pathological events occurring at the mitochondrial level during the "critical period" in women and men. During the reproductive life (1), high level of sex hormones, represented on a second axis on the top (women) and at the bottom (men) of the graphic, are paralleled by an "normal" mitochondrial function with low levels of reactive oxygen species (ROS). At the beginning of the reproductive senescence (2), the decrease of sex hormones is coupled with an increased oxidative stress. ROS produced by mitochondria can eventually surpass a pathological threshold (dashed line) leading to $A \beta$ accumulation, 
tau hyperphosphorylation and neuroinflammation, which can in turn affect mitochondrial function. Fortunately, cells possess compensatory mechanisms (antioxidant defenses, $A \beta$ degrading enzymes...) which can keep the system in balance, leading to normal "nonpathological aging" (3). In case the system is overwhelmed (3'), failure of compensatory mechanisms may lead to deep metabolic impairments, leading to neuronal death and AD. As indicated by the square (at the top), this "critical period", in which sex hormones are decreasing, occurs earlier and within a shorter period of time in women compared to men (square in dashed line at the bottom). This might explain why women are more vulnerable and more likely to develop AD at this period of life (see details in the text). 